\title{
CARDIO-ONCOLOGY - THE NEW KID ON THE BLOCK
}

As the life expectancy is improving, the risk of both cardiovascular disease and cancer is increasing. Cancer and cardiovascular disease are the leading causes of morbidity and mortality worldwide. Therefore, it is no surprise that most cancer centres are now inundated with an ageing population of cancer patients who also have multiple cardiac issues. Over the past few decades, there has been significant improvement in the quality of life and survival of cancer patients due to the introduction of newer therapies. Chemotherapy seems to have been pushed to the backstage, with targeted therapy now in the forefront. Advances in molecular therapy monoclonal antibodies and immunotherapy are changing the landscape for cancer patients. As the life expectancy is improving due to advances in cardiovascular medicine, the incidence of cancer is rising in the ageing population.

It is important to note the fact that both cancer and cardiovascular disease have somewhat shared origins. The major cardiovascular risk factors such as obesity cigarette smoking poor dietary habits lack of exercise alcoholism and increasing age are shared by both disease entities. Similarly, due to the intense nature of cancer therapy, patients on active treatment tend to develop unhealthy lifestyles including physical inactivity excessive weight loss or weight gain high levels of stress and anxiety, all of which enhance the risk of cardiovascular morbidity and mortality.

Cancer therapy associated cardiotoxicity is a well-known phenomenon. Association between anthracycline use and cardiotoxicity was identified almost five decades ago. ${ }^{[1]}$ Cardiovascular mortality has been reported as the main cause of mortality 10 years after the diagnosis of breast cancer. ${ }^{[2]}$ Over the years, however, the development of new targeted therapies has resulted in a wide variety of cardiac problems. The term 'cancer therapeutics related cardiac dysfunction' has been used to define the potential cardiotoxicity in such instances. ${ }^{[3]}$ This knowledge has

Correspondence: Dr. Ahmed Awais, MD,

Medical Oncology Department, Shaukat Khanum Memorial Cancer

Hospital and Research Centre, Lahore, Pakistan.

Email: ahmedawais@skm.org.pk certainly led to better outcomes and has given rise to a novel cardiology subspecialty known as cardio-oncology, with the international cardiology oncology society having been established in 2009 .

Cardiotoxicity may be attributed to the direct effect of cancer therapy on the heart, but it could also be due to the development or unmasking of underlying cardiovascular diseases in cancer patients. Within the speciality of cardio-oncology, years of research and clinical practice have given birth to the multiple hit hypothesis, which was first described in 2007. ${ }^{[4]}$ This hypothesis proposes that patients with cancer are exposed to a series of sequential or concurrent events that together make them more vulnerable to reduced cardiovascular reserves the development of incidental cardiovascular disease and eventually death.

Cardiotoxicity can be of various types. Since most cancer centres have a large volume of breast cancer and lymphoma patients, anthracycline-associated cardiotoxicity has been the most frequently encountered problem in the past. Other than anthracycline agents such as trastuzumab cyclophosphamide and tyrosine kinase inhibitors have been shown to cause left ventricular dysfunction. While anthracycline-induced cardiotoxicity is permanent due to destruction of myocytes, trastuzumab-associated dysfunction is usually reversible. ${ }^{[5,6]}$ Hypertension is also a well-known sequela of treatment with vascular endothelial growth factor inhibitors such as bevacizumab sorafenib sunitinib and also erythropoietin-stimulating agents. ${ }^{[7,8]}$ Certain drugshave been implicated in predisposition to cardiac arrhythmias such as atrial fibrillation and QT interval prolongation. Medications used for supportive care in cancer patients such as antiemetics antidepressants antihistamines and antipsychotics can also lead to cardiac conduction disturbances. Chemotherapy drugs like 5 -fluorouracil have a well-known association with coronary vasospasm and angina-like symptoms. Proteasome inhibitors have also been implicated in cardiac morbidity. More recently, the use of checkpoint inhibitor therapy has resulted in rare, but serious cardiotoxicity including myocarditis cardiac arrest and maybe even myocardial fibrosis. ${ }^{[9]}$ Radiation treatment, which is often employed in cancer treatment, can also lead 
to accelerated atherosclerosis pericardial inflammation or damage to the heart valves. ${ }^{[10]}$

Given the myriad of cardiac issues associated with cancer treatments, it is very important to establish cardio-oncology services in large volume cancer centres. Knowledge of the adverse effects of cancer therapy on the heart balanced with knowledge of the natural history of malignancy offers an opportunity to provide effective care to the cancer patients. The cardio-oncology team is a multidisciplinary team of professionals who provide care with a special focus on cardiovascular disease. Such a team comprises medical and radiation oncologists haematologists cardiologists and specialised nurses. Supporting members may include hospitalists surgeons pathologists radiologists pharmacists psychologists social workers and members of the palliative care team. The medical oncologists are expected to refer patients to the cardiology clinic in a timely fashion. The role of the cardiologist is to facilitate cancer treatment.

Large volume cancer centres in the United States have set up cardio-oncology clinics with the goal of facilitating the diagnosis and therapy of cancer treatment-related cardiovascular complications. One of the reputable centres in the United States initially utilized a computerbased consultation system, and later, face-to-face clinical encounters were set up for cancer patients needing dedicated cardiac care. Educational sessions for both health-care staff and patients were set up. This clinic witnessed healthy growth over a short period of time, with promising results expected in the long run. Interdisciplinary communication was found to be essential for the effective operation of this cardio-oncology unit. ${ }^{[11]}$

In the day-to-day functioning of the cardio-oncology unit, it is imperative to identify individuals who are at high risk for cardiotoxicity, such as those with poor lifestyle habits, or comorbidities such as hypertension coronary artery disease and diabetes. This screening includes not only patients who are receiving potentially cardiotoxic chemotherapy but also those who are to undergo any cancer-related surgery. Such patients should have baseline blood pressure measurement along with an electrocardiogram or echocardiogram as deemed appropriate. Baseline assessment of QT interval is relevant in certain patients. Similarly, an echocardiogram is essential for those who will be subjected to anthracyclines or Her-2 targeted therapy. As we know from prior studies, there is a sudden rise in the probability of heart failure once the cumulative dose of anthracycline exceeds $400 \mathrm{mg} / \mathrm{m}^{2}$. There are no sensitive echocardiographic techniques such as strain imaging that can detect subtle left ventricular dysfunction. For example, a decrease in the global longitudinal strain of $>15 \%$ from baseline is a sign of early cardiotoxicity. ${ }^{[12]}$ Similarly, biomarkers such as plasma troponin concentration or B-type natriuretic peptide can be used as indicators of early cardiotoxicity. ${ }^{[13]}$

Once baseline evaluation has been performed, patients should have active surveillance of their cardiovascular health during the treatment. This includes echocardiography, cardiac MRI, or any other pertinent studies. Any changes in cardiovascular status should immediately prompt the team to reevaluate the patient's treatment plan. The need for cardioprotective medical therapy, for example, the use of dexrazoxane to combat anthracycline-mediated cardiotoxicity or the use of beta-blockers angiotensin-converting enzyme inhibitors or statins in patients with a subclinical drop in ejection fraction should be considered on a case-by-case basis. ${ }^{[13,14]}$

Once a cardio-oncology clinic has been set up, audits can be conducted from time to time to test the efficiency of the system and to make sure that there are no unnecessary delays in the process of referral. Computer software can be used to maintain a separate database of such patients. Finally, collection of patient feedback through questionnaires or surveys is helpful in identifying any weaknesses and deficiencies in the program. ${ }^{[15]}$

In conclusion, it should be a top priority of oncologists and haematologists to work in conjunction with their cardiology colleagues to evaluate the cardiovascular health of their patients and to address the potential cardiotoxicity associated with cancer therapy. Setting up a cardio-oncology program seems to be the best way to achieve this objective. Comprehensive cancer centres in the United States and Europe have now set up dedicated cardio-oncology clinics, and many other centres are now following suit. The creation of such a partnership will undoubtedly lead to a high-quality medical practice and will ensure the provision of multidisciplinary care to our cancer patients. 


\section{Ahmed Awais ${ }^{1}$, Irfan A. Vaziri², Benjamin George ${ }^{3}$}

${ }^{1}$ Department of Medical Oncology, Shaukat Khanum Memorial Cancer Hospital and Research Centre, Lahore, Pakistan, ${ }^{2}$ Callahan Cancer Center Nebraska, USA, ${ }^{3}$ Banner MD Anderson, Colorado, USA

Received: 02 September 2018 / Accepted: 17 September 2018

\section{References}

1. Von Hoff DD, Layard MW, Basa P, et al. Risk factors for doxorubicin-induced congestive heart failure. Ann Intern Med 1979;91:710-7.

2. Patnaik JL, Byers T, DiGuiseppi C, et al. Cardiovascular disease competes with breast cancer as the leading cause of death for older females diagnosed with breast cancer: A retrospective cohort study. Breast Cancer Res 2011;13:R64.

3. Plana JC, Galderisi M, Barac A, et al. Expert consensus for multimodality imaging evaluation of adult patients during and after cancer therapy: A report from the American society of echocardiography and the European association of cardiovascular imaging. Eur Heart J Cardiovasc Imaging 2014; 15:1063-93

4. Jones LW, Haykowsky MJ, Swartz JJ, et al. Early breast cancer therapy and cardiovascular injury. J Am Coll Cardiol 2007;50:1435-41.

5. Tan TC, Neilan TG, Francis S, et al. Anthracycline-induced cardiomyopathy in adults. Compr Physiol 2015;5:1517-40.

6. Gorini S, De Angelis A, Berrino L, et al. Chemotherapeutic drugs and mitochondrial dysfunction: Focus on doxorubicin, trastuzumab, and sunitinib. Oxid Med Cell Longev 2018;18:7582730.

7. Berardi D, Agati L. Cardiovascular adverse reactions after the administration of recombinant human erythropoietin: Light and shade. Minerva Cardioangiol 2012;60:227-36.

8. Yeh ET, Bickford CL. Cardiovascular complications of cancer therapy: Incidence, pathogenesis, diagnosis, and management. J Am Coll Cardiol 2009;53:2231-47.

9. Mahmood SS, Fradley MG, Cohen JV, et al. Myocarditis in patients treated with immune checkpoint inhibitors. J Am Coll Cardiol 2018;71:1755-64.

10. Lancellotti P, Nkomo VT, Badano LP, et al. Expert consensus for multi-modality imaging evaluation of cardiovascular complications of radiotherapy in adults: A report from the European association of cardiovascular imaging and the American society of echocardiography. Eur Heart J Cardiovasc Imaging 2013;14:721-40.

11. Barros-Gomes S, Herrmann J, Sharon L, et al. Rationale for setting up a cardio-oncology unit: Our experience at mayo clinic. Cardio Oncol 2016;2:5.

12. Zamorano JL, Lancellotti P, Rodriguez Muñoz D, et al. 2016 ESC position paper on cancer treatments and cardiovascular toxicity developed under the auspices of the ESC committee for practice guidelines: The task force for cancer treatments and cardiovascular toxicity of the European society of cardiology (ESC). Eur Heart J 2016;37:2768-801.

13. Kostakou PM, Kouris NT, Kostopoulos VS, et al. Cardio-oncology: A new and developing sector of research and therapy in the field of cardiology. Heart Fail Rev 2019;24:91-100.

14. Sharalaya Z, Collier P. Prevention of cardiotoxicities with traditional and novel chemotherapeutic agents. Curr Heart Fail Rep 2018;15:260-9.

15. Lancellotti P, Suter TM, López-Fernández T, et al. Cardio-oncology services: Rationale, organization, and implementation: A report from the ESC cardio-oncology council. Eur Heart J 2018. Doi: 10.1093/eurheartj/ehy453. 